\title{
Prevalence of the Human Papilloma Virus (HPV) genotypes in south-central Sicily: a comparative study between 2003 and 20 I |
}

\author{
Liborio Bellomo, Rosalba Collodoro, Giuseppina Di Forti, Claudia Lo Magno \\ U.O.Virologia Asp n.2 Caltanissetta

\begin{abstract}
Prevalenza dei genotipi di Papilloma Virus Umano (HPV) nella Sicilia centro-meridionale: studio comparativo fra il 2003 e il 201 I
\end{abstract}

Key words: genotype, HPV, prevalence

\section{SUMMARY}

This issue is about our Human Papilloma Virus (HPV) DNA test.

We have examined two cohorts of patients from south-central Sicily, who were tested for HPV -DNA derived from cervical sampling, respectively in 2003 and $201 \mathrm{I}$.

In 2003, the most represented genotypes were: 31, 16. Instead, in 201 I there was a higher prevalence for genotypes: 42 , 16. It is remarkable to note that the 35 genotype, noticed in 2003, has never been found in the second most recent group.

\section{INTRODUZIONE}

I Papillomavirus sono associati a lesioni proliferative, benigne e maligne, della cute e delle mucose. Le infezioni possono essere subcliniche, cliniche o latenti. Il virus ha un tropismo molto ristretto e replica nelle cellule epiteliali in differenziamento di cute e mucose. In letteratura è riportato che le neoplasie sincrone in siti genitali plurimi (cervice, vagina, vulva, perineo, etc) sono spesso correlate all'azione di Human Papilloma Virus (HPV) genotipo 16: normalmente il processo di progressione verso la malignità si attua lentamente, assicurando così margini temporali di intervento.

Si è riscontrato che sovente $i$ componenti di una coppia sono portatori degli stessi genotipi di HPV. Infezioni dell'area anogenitale sono più frequenti tra i maschi omosessuali spesso colpiti ad esempio da verruche distrettuali.

HPV vengono classificati in genotipi ad alto e basso rischio in relazione alla loro oncogenicità ed alla frequenza con cui si riscontrano a livello delle lesioni genitali (Tabella 1) $(2,10)$.

Tale attività si correla alla capacità del virus di integrare il proprio genoma in quello della cellula ospite e successivamente interagire con i delicati meccanismi di replicazione cellulare.

La trasmissione avviene per contatto diretto con una persona infetta, per autoinoculazione o per contatto indiretto. Le verruche genitali presentano una trasmissione sessuale e si stima un'incidenza tra le donne, proporzionale al numero dei partner sessuali, variabile dal 2-13\% in Europa occidenta- le a più del $30 \%$ in America Latina (5). Nelle giovani donne siciliane, secondo alcuni Autori si è rilevata una maggiore prevalenza dei genotipi 16 e 53 (1) a livello della cervice uterina. In circa il $90 \%$ dei casi di neoplasia intraepiteliale della cervice uterina si è rilevata la presenza del materiale genetico del virus.

Si è osservato che HPV-6 e HPV-11 sono responsabili di affezioni benigne del tratto respiratorio e digestivo e HPV-16 è correlato ad alcune affezioni maligne (papillomatosi respiratoria, CA dell'ultima porzione dell'intestino, etc); la localizzazione più frequente è alle corde vocali, ma sono coinvolti anche trachea, polmone, naso, cavità orale. Altre affezioni quali Leucoplachia orale (OL) e Lichen ruber planus (OLP) sono correlate alla presenza di HPV-18, HPV-16, HPV -33, HPV-31 e HPV-6; co-fattori quali fumo, alcool e l'età aumentano il rischio neoplastico (3).

$\mathrm{Nel}$ giugno del 2006 il vaccino quadrivalente dal nome GARDASIL/SILGARD ${ }^{\mathbb{R}}$ (Merck, NJ, USA) è stato autorizzato in USA (7). Alcuni studi dimostrano che HPV è in grado di inibire la secrezione di IFN-g e l'espressione dei recettori cellulari di alcune cellule immunocompetenti, in particolare ILT2. Studi effettuati dopo vaccinazione con GARDASIL hanno dimostrato un aumento significativo dell'espressione dei recettori ILT2 di NK e dei linfociti CD3+ e CD56+ (4).

La Sicilia è stata tra le prime regioni ad intraprendere una campagna vaccinale gratuita, già nel 2007/08, per le ragazzine di 11 anni, mentre le

\section{Corresponding author: Liborio Bellomo}

Via L. Russo 6, 93100 Caltanissetta

Tel.: 093455923 I - Fax 0934559555

E-mail: liboriobellomo@virgilio.it 
donne adulte potevano sottoporsi alla vaccinazione volontariamente anche a costi agevolati (6).

Il vaccino esplica la sua azione protettiva nei confronti dei genotipi responsabili della maggior parte dei carcinomi cervicali (HPV16-18) e delle lesioni proliferative benigne (HPV6-11), ma non riesce a garantire la copertura nei confronti di tutti i tipi di HPV. Non si conoscono gli effetti della copertura vaccinale a distanza di anni. È auspicabile, quindi, che le donne continuino a sottoporsi successivamente ad accertamenti diagnostici. Comunque, studi effettuati su maschi di età compresa fra 16-26 anni riportano dati significativi sulla effettiva protezione da parte del vaccino GARDASIL nei confronti dell'infezione (8).

Il vaccino presenta una buona immunogenicità e sicurezza anche in pazienti affetti da Lupus eritematoso sistemico (9).

Con il presente studio si è voluto confrontare le prevalenza dei genotipi di HPV nel 2011 con il kit di biologia molecolare attualmente in uso presso la U.O. di Virologia del Presidio Ospedaliero di Caltanissetta, con quelli del 2003 con il kit allora utilizzato (anno di inizio dell'esecuzione del test molecolare), al fine di valutare eventuali differenze che potrebbero essere influenzate da numerose variabili.

\section{MATERIALI E METODI}

Il bacino di utenza dell'Unità Operativa di Virologia del Presidio Ospedaliero S. Elia di Caltanissetta è costituito da Caltanissetta, Enna, Agrigento e relative provincie.

Nell'anno 2003 sono stati esaminati 211 tamponi cervico-vaginali per la ricerca del materiale genomico di HPV. Le pazienti erano state inviate dai medici di medicina generale, dagli specialisti territoriali e dalle strutture ospedaliere. I campioni sono stati analizzati con il kit Geno-Kit HPV L1 "generale" (AMPLIMEDICAL, Torino, Italy).

Negli anni il numero delle utenti è aumentato fino a giungere nel 2011 i 404 campioni, i quali sono stati testati con il kit "LINEAR ARRAY HPV Genotipyng Test" (Roche Diagnostics, Mannheim, Germany).

Il kit utilizzato nel 2003 prevedeva l'utilizzo di sonde specifiche marcate con biotina per i genotipi di HPV 6, 11, 16, 18, 31, 33, 35, 45. L'ibridazione delle sonde con i prodotti di amplificazione era evidenziata attraverso il legame streptavidina-perossidasi e conseguente reazione colorimetrica con il cromogeno 3.3'-5.5'-tetrametilbenzidina (TMB).

Il kit "LINEAR ARRAY HPV Genotipyng Test" utilizzato nel 2011 prevede una iniziale amplificazione con successiva reverse hybridation $(\mathrm{RH})$ ed è in grado di identificare un maggior numerodi genotipi: $6,11,16,18,26,31,33,35,39,40,42$, $43,44,45,51,52,53,54,55,56,57,58,59,61$, $62,64,66,67,68,69,70,71,72,73,81,82,83$, 84, CP6108, IS39.

\section{RISULTATI}

Nel 2003 sono stati analizzati 211 tamponi: il $24.17 \%$ sono risultati positivi per HPV, il $73.46 \%$ negativi e il $2.37 \%$ non testabili (Figura I).

La percentuale dei genotipi sul totale dei campioni varia dallo $0.95 \%$ del genotipo 6 al $7.11 \%$ del genotipo 31 (Tabella 2 e Figura II). Considerando solo i campioni positivi, le percentuali dei genotipi sono riportate in tabella 2 e figura III.

Negli anni, il numero degli utenti è aumentato fino a raggiungere nel 2011 il numero di 400 campioni. Con l'utilizzo di un kit, in grado di rivelare un maggior numero di genotipi rispetto al 2003, nel 2011 il 58.50\% dei campioni è risultato positivo, il $40.25 \%$ negativo e $1 ' 1.25 \%$ non testabile (Figura IV).

Le percentuali dei genotipi sul totale dei campioni e dei soli campioni positivi, sono riportate in tabella 3 e rispettivamente in figura V e VI.

Il confronto dei risultati ottenuti per i genotipi più comuni ottenuti nel 2003 e 2011 sono riportati in figura VII e VIII.

\section{DISCUSSIONE E CONCLUSIONI}

Dai nostri dati risulta che nel 2003 i genotipi più presenti erano $31(29.41 \%), 16(25.49 \%)$ e 35 (13.72\%) mentre nel 2011 erano 42 (11.54\%), 16 (8.55\%), $31(6.84 \%)$ e $54(6.41 \%)$.

Mentre i genotipi 16 e 31 sono sempre presenti nei due gruppi (anche se con percentuali diverse), il genotipo 35 non è stato mai riscontrato nel gruppo dei pazienti più recenti. Inoltre il genotipo 6, implicato in patologie benigne, non ha mostrato notevoli variazioni percentuali.

Le differenze riscontrate tra i due gruppi possono essere dovute a vari fattori: sicuramente

la percentuale di campioni non classificabili del 2003 è legata alla ridotta capacità del kit utilizzato di discernere solo pochi genotipi rispetto a quelli identificabili con il kit in uso nel 2011. Inoltre la selezione dei pazienti candidati al test è verosimilmente migliorata nel tempo con una numerosità della campionatura nel 2011 circa il doppio di quella del 2003.

Anche l'introduzione della vaccinazione per HPV può aver influenzato la diffusione dei vari genotipi. Infine, la quota di campioni non testabili del 2011 è verosimilmente da ricondurre ad un problema tecnico verificatosi durante l'esecuzione del prelievo; questa ipotesi è suffragata dal fatto che la ripetizione del prelievo ha consentito di ottenere un risultato definito. 
È da sottolineare che la percentuale dei negativi ai test è scesa da 73.46\% nel 2003 al $40.25 \%$ nel 2011, ma è comunque consistente, e quindi è auspicabile ottenere migliori risultati per il futuro anche implementando progetti a ciò dedicati.

Date le diverse performances dei due test utilizzati, ci si propone di eseguire nel prossimo futuro un ulteriore studio utilizzando lo stesso test del 2011.

Tabella I. Genotipi HPV ad alto e basso rischio in relazione alla loro oncogenicità.

\begin{tabular}{cc}
\hline INDICE DI RISCHIO & GENOTIPI \\
\hline Alto rischio & $|6| 8,26,,3 I, 33,35,39,45,5 I, 52,53,56,58,59,66,68,73,82$ \\
\hline Basso rischio & $6, I I, 40,42,54,55,6 I, 62,64,67,69,70,7 I, 72,8 I, 83,84, I S 39$, CP6I08 \\
\hline
\end{tabular}

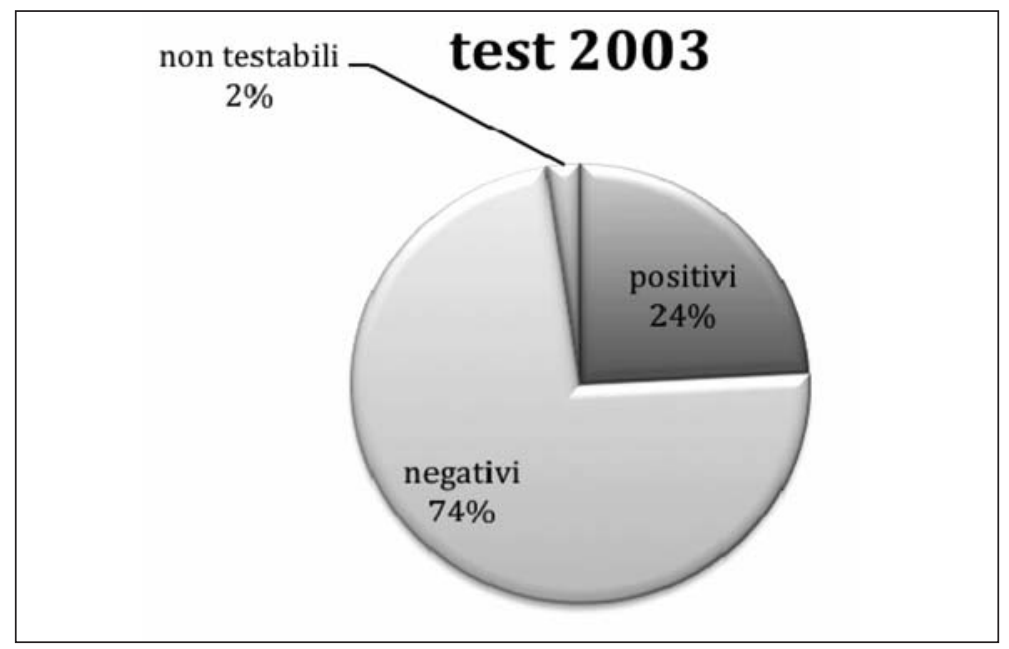

Figura I. Distribuzione dei risultati per ricerca dei genotipi HPV nel 2003 su 2 I I tamponi cervico-vaginali.

Tabella 2. Distribuzione genotipi HPV nel 2003

\begin{tabular}{cccc}
\hline Genotipo HPV & $\begin{array}{c}\text { Numero positivi } \\
\text { per genotipo }\end{array}$ & $\begin{array}{c}\text { Percentuale genotipo } \\
\text { sul totale }(21 \mathrm{I})\end{array}$ & $\begin{array}{c}\text { Percentuale genotipo } \\
\text { sul totale positivi (5I) }\end{array}$ \\
\hline 6 & 2 & $0.95 \%$ & $3.92 \%$ \\
\hline 11 & 3 & $1.42 \%$ & $5.88 \%$ \\
\hline 16 & 13 & $6.16 \%$ & $25.49 \%$ \\
\hline 18 & 5 & $2.37 \%$ & $9.80 \%$ \\
\hline 31 & 15 & $7.11 \%$ & $29.41 \%$ \\
\hline 35 & 7 & $3.31 \%$ & $3.72 \%$ \\
\hline 45 & 2 & $0.94 \%$ & $7.92 \%$ \\
\hline Non classificabile & 4 & $1.89 \%$ & $7.84 \%$ \\
\hline
\end{tabular}




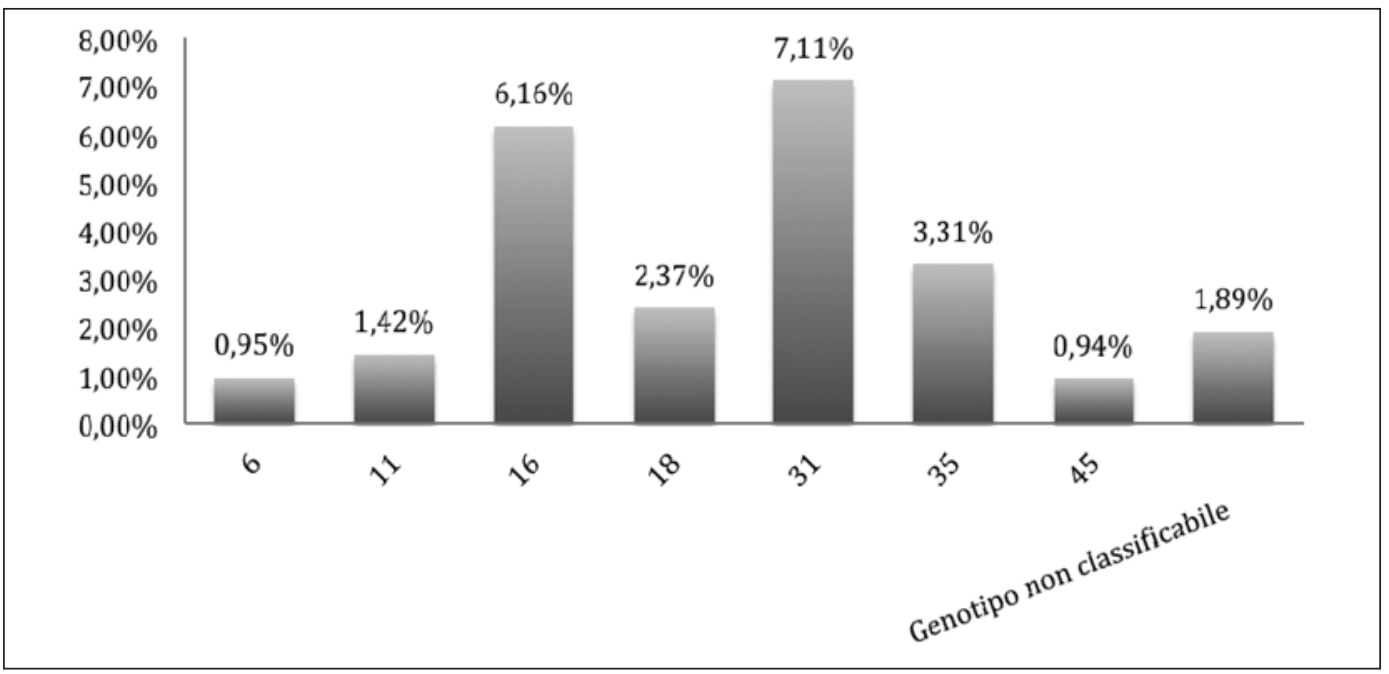

Figura II. Percentuale dei genotipi HPV espressi sul totale dei campioni analizzati nel 2003.

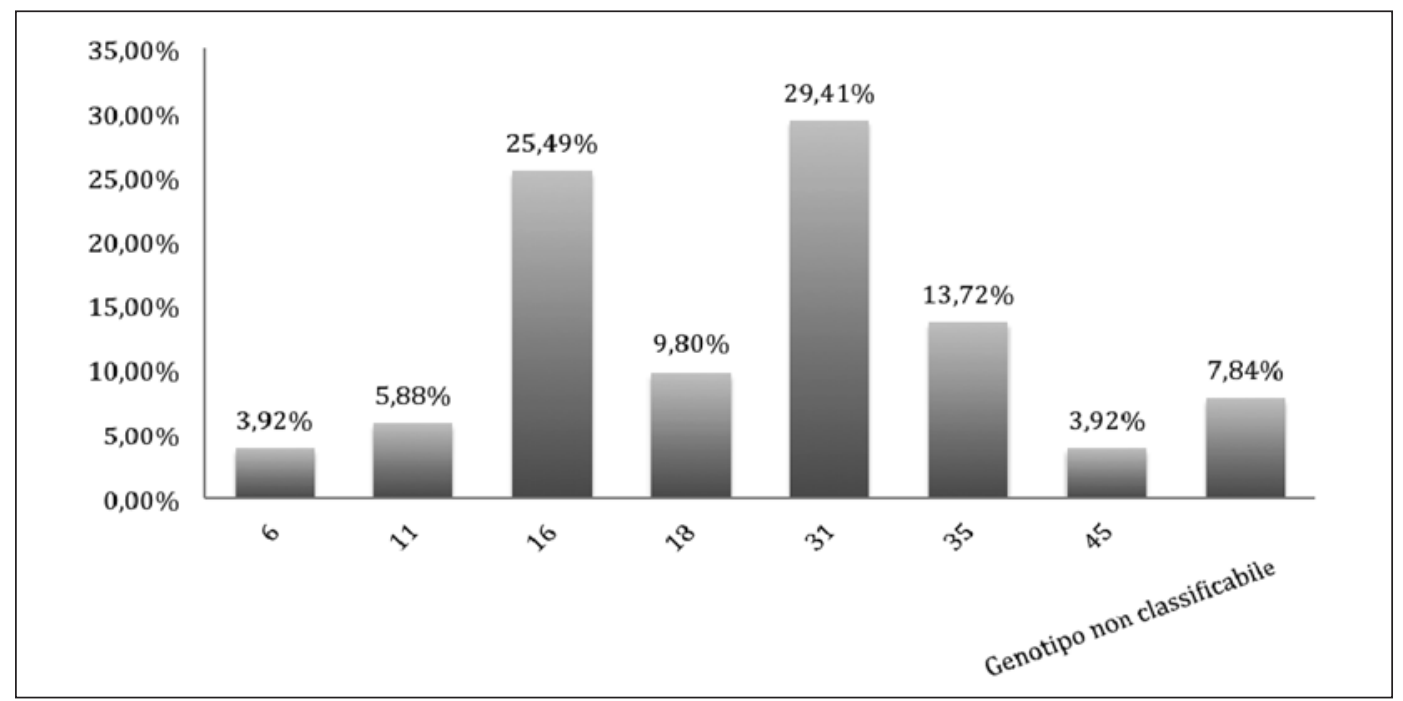

Figura III. Percentuale dei genotipi HPV espressi sul totale dei positivi analizzati nel 2003.

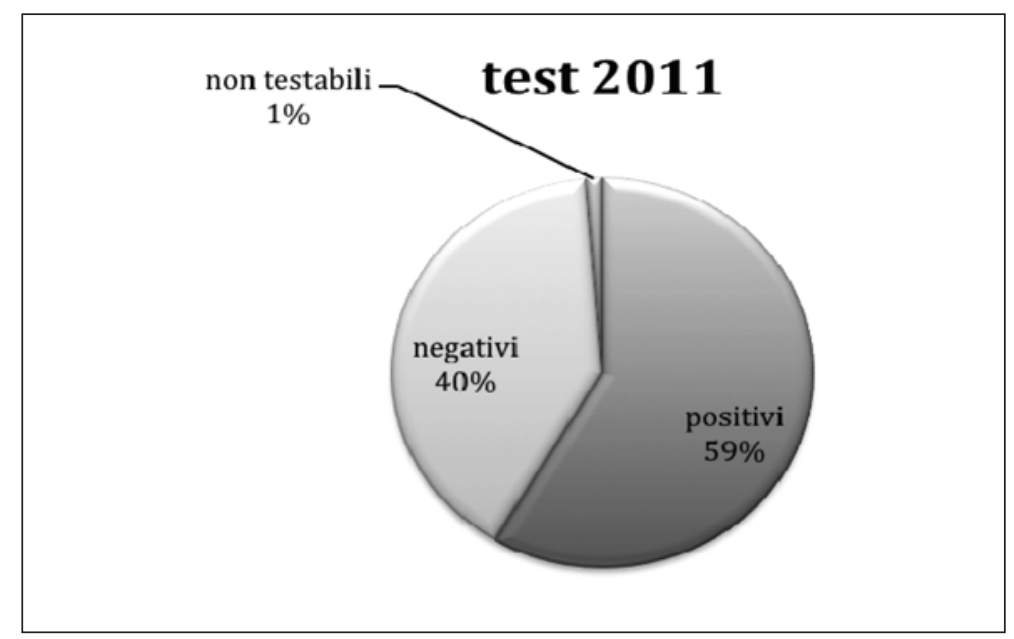

Figura IV. Distribuzione dei risultati per ricerca dei genotipi HPV nel 20 I I su 400 tamponi cervico- vaginali. 
Tabella 3. Distribuzione dei genotipi HPV nel 20II.

\begin{tabular}{|c|c|c|c|}
\hline Genotipo & $\begin{array}{l}\text { Numero positivi } \\
\text { per genotipo }\end{array}$ & $\begin{array}{l}\text { Percentuale genotipo } \\
\text { sul totale }(400)\end{array}$ & $\begin{array}{l}\text { Percentuale genotipo } \\
\text { sul totale positivi (234) }\end{array}$ \\
\hline 6 & 10 & $2.50 \%$ & $4.27 \%$ \\
\hline II & 5 & $1.25 \%$ & $2.14 \%$ \\
\hline 16 & 20 & $5.00 \%$ & $8.55 \%$ \\
\hline 18 & 4 & $1.00 \%$ & $1.71 \%$ \\
\hline 31 & 16 & $4.00 \%$ & $6.84 \%$ \\
\hline 33 & $\mathrm{I}$ & $0.25 \%$ & $0.43 \%$ \\
\hline 39 & 5 & $1.25 \%$ & $2.14 \%$ \\
\hline 40 & 1 & $0.25 \%$ & $0.43 \%$ \\
\hline 42 & 27 & $6.75 \%$ & $11.54 \%$ \\
\hline 45 & 8 & $2.00 \%$ & $3.42 \%$ \\
\hline 51 & 4 & $1.00 \%$ & $1.71 \%$ \\
\hline 52 & 11 & $2.75 \%$ & $4.70 \%$ \\
\hline 53 & 9 & $2.25 \%$ & $3.85 \%$ \\
\hline 54 & 15 & $3.75 \%$ & $6.41 \%$ \\
\hline 55 & 1 & $0.25 \%$ & $0.43 \%$ \\
\hline 6 & 4 & $1.00 \%$ & $1.71 \%$ \\
\hline 58 & 10 & $2.50 \%$ & $4.27 \%$ \\
\hline 59 & 8 & $2.00 \%$ & $3.42 \%$ \\
\hline 61 & 4 & $1.00 \%$ & $1.71 \%$ \\
\hline 62 & 6 & $1.50 \%$ & $2.56 \%$ \\
\hline 66 & 9 & $2.25 \%$ & $3.85 \%$ \\
\hline 67 & $\mathrm{I}$ & $0.25 \%$ & $0.43 \%$ \\
\hline 68 & 5 & $1.25 \%$ & $2.14 \%$ \\
\hline 70 & $\mathrm{I}$ & $0.25 \%$ & $0.43 \%$ \\
\hline 72 & $\mathrm{I}$ & $0.25 \%$ & $0.43 \%$ \\
\hline 73 & 14 & $3.50 \%$ & $5.98 \%$ \\
\hline 81 & 4 & $1.00 \%$ & $1.71 \%$ \\
\hline 82 & $\mathrm{I}$ & $0.25 \%$ & $0.43 \%$ \\
\hline 83 & 2 & $0.50 \%$ & $0.85 \%$ \\
\hline 84 & 14 & $3.50 \%$ & $5.98 \%$ \\
\hline CP6I08 & 13 & $3.25 \%$ & $5.55 \%$ \\
\hline
\end{tabular}

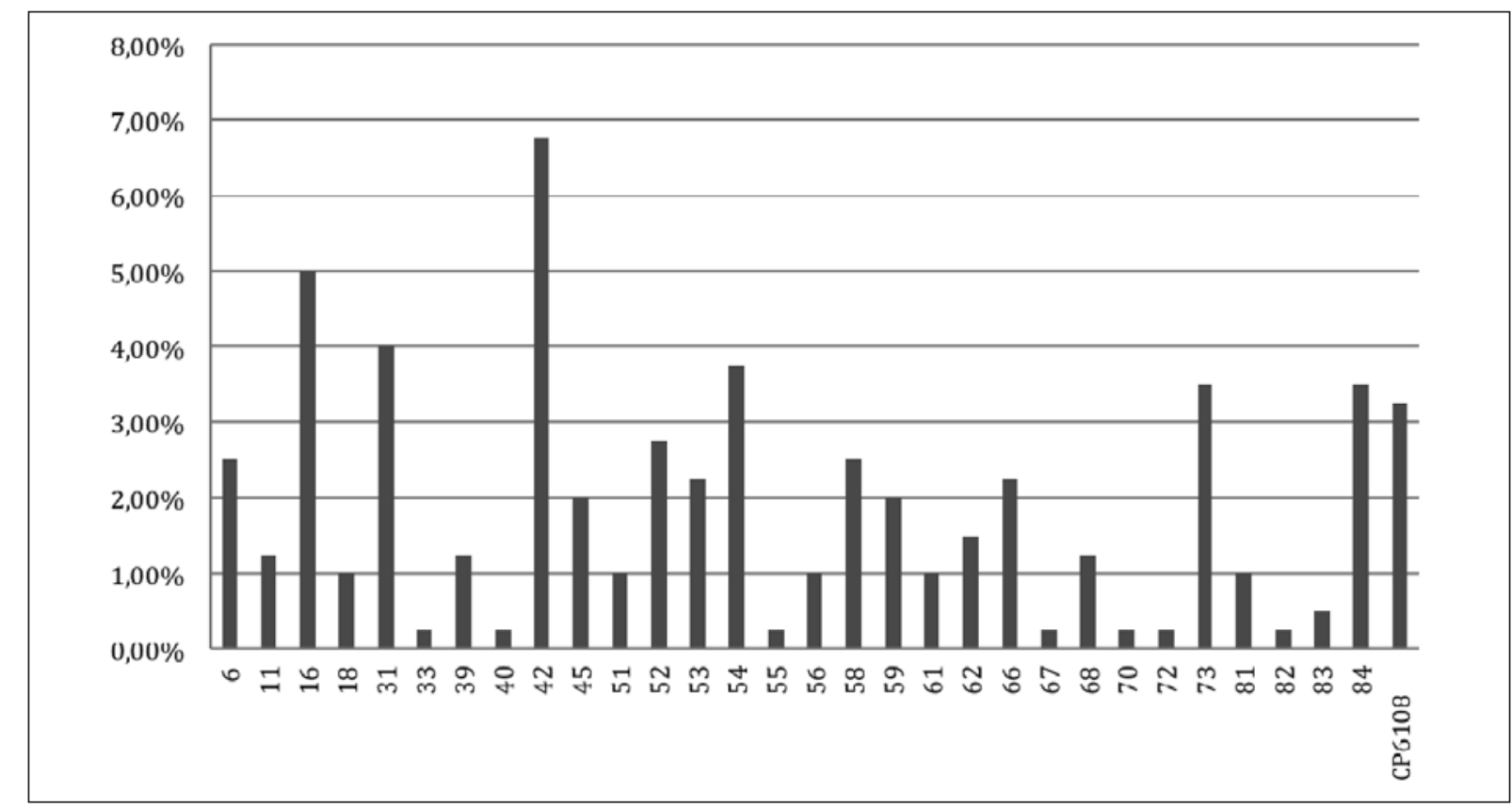

Figura V. Percentuale dei genotipi HPV sul totale dei campioni analizzati nel 201 I. 


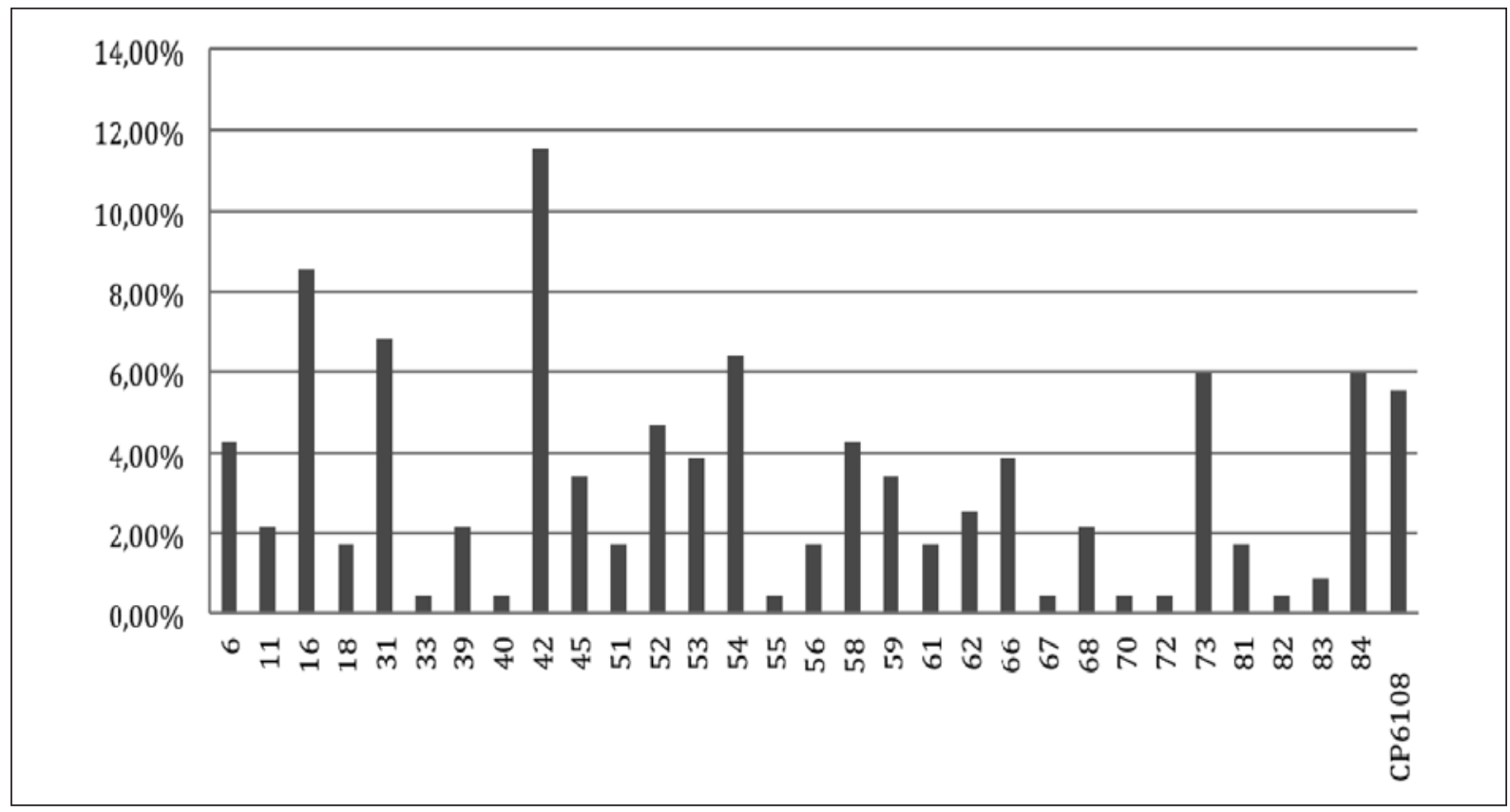

Figura VI. Percentuale dei genotipi HPV sul totale dei positivi analizzati nel 201 I.

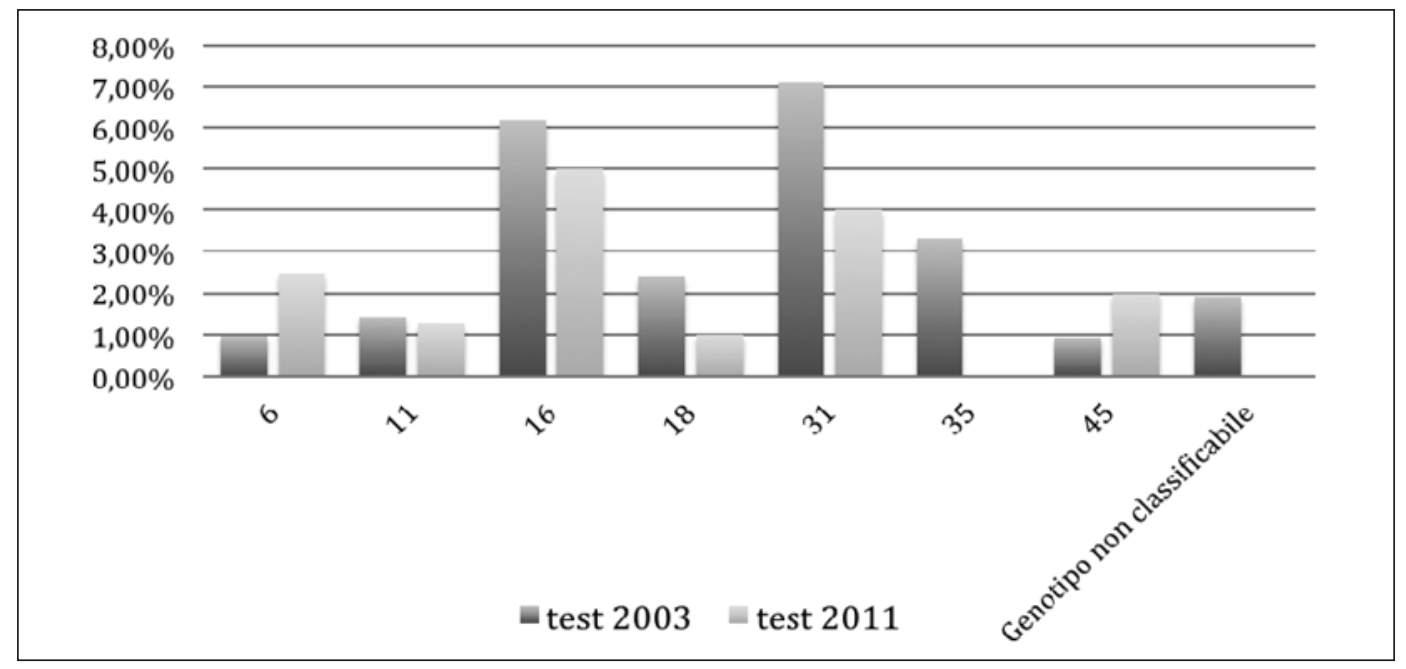

Figura VII. Confronto dei risultati ottenuti nel 2003 e nel 201 I per genotipi HPV sul totale dei campioni analizzati.

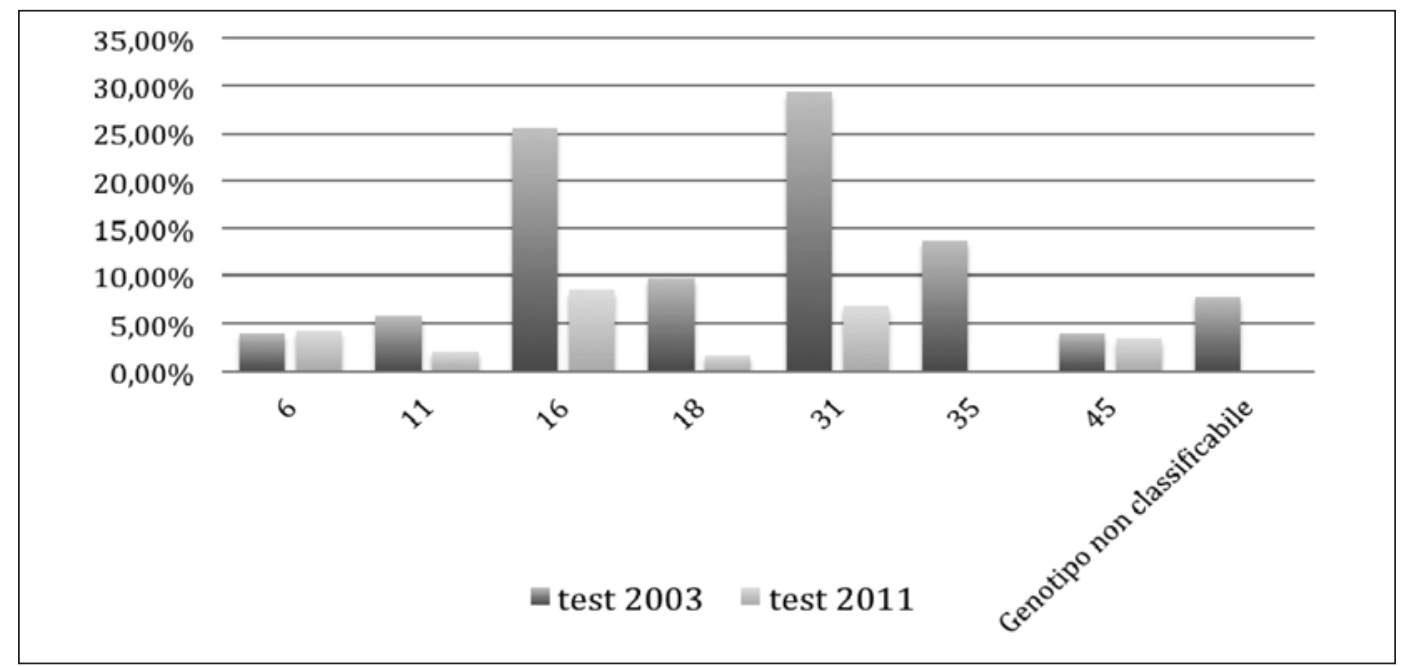

Figura VIII. Confronto dei risultati ottenuti nel 2003 e nel 201 I per genotipi HPV sul totale dei campioni positivi. 


\section{BIBLIOGRAFIA}

1. Ammatuna $\mathrm{P}$, Giovannelli L, Matranga $\mathrm{D}$, et al. Prevalence of genital human papilloma virus infection and genotypes among young women in Sicily, South Italy. Cancer Epidemiol Biomarkers Prev 2008; 17: 2002-6.

2. Calleja-Macias IE, Villa LL, Prado JK, et al. Worldwide genomic diversity of the high-risk human papillomavirus types $31,35,52$, and 58 , four close relatives of human papillomavirus type 16 . J Virol 2005; 79: 13630-40.

3. Campisi G, Giovannelli L, Aricò P, et al. HPV DNA in clinically different variants of oral leukoplakia and lichen planus. Oral Surg Oral Med Oral Pathol Oral Radiol Endod 2004; 98: 705-11.

4. Colmenares V, Noyola DE, Monsiváis-Urenda A, et al. Human papilloma virus (HPV) immunization is associated with increased expression of different innate immune regulatory receptors. Clin Vaccine Immunol 2012; 19: 1005-11.

5. Dianzani F, Antonelli G, Capobianchi MR, Dolei A, eds. Manuale di Virologia Medica. 3rd edn. Milano:
McGraw-Hill, 2008: 343-4.

6. Gazzetta Ufficiale Regione Sicilia (GURS). Avvio della campagna vaccinale anti-HPV in Sicilia. Decreto assessoriale del 29/02/2008 parte $1 \mathrm{n} .14$.

7. Goldstone SE, Vuocolo S. A prophylactic quadrivalent vaccine for the prevention of infection and disease related to HPV-6, -11, -16 and -18. Expert Rev Vaccines 2012; 11: 395-406.

8. Hillman RJ, Giuliano AR, Palefsky JM, et al. Immunogenicity of the quadrivalent human papillomavirus (type 6/11/16/18) vaccine in males 16 to 26 years old. Clin Vaccine Immunol 2012; 19: 261-7.

9. Mok CC, Ho LY, Fong LS, To CH. Immunogenicity and safety of a quadrivalent human papillomavirus vaccine in patients with systemic lupus erythematosus: a case-control study. Ann Rheum Dis 2012 May 15 [Epubahead of print].

10. Muñoz N, Bosch FX, de Sanjosé S, et al. for the International Agency for Research on Cancer Multicenter Cervical Cancer Study Group. Epidemiologic classification of human Papillomavirus types associated with cervical cancer. $N$ Engl J Med 2003; 348: 518-27. 